Thorax (1946), 1, 247.

\title{
SUPERIOR VENA CAVAL OBSTRUCTION DUE TO CHRONIC MEDIASTINITIS
}

\author{
BY \\ OSWALD S. TUBBS \\ St. Bartholomew's Hospital, London
}

Obstruction of the superior vena cava due to chronic mediastinitis has been recognized for at least a century (Hallet, 1848); and Ochsner and Dixon (1936) in their review of 120 reported cases of thrombosis of the superior vena cava found that 28 of these were caused by a fibrotic process within the mediastinum.

Two such cases have come under my care during the last six years and I have had the privilege of seeing a third at the invitation of Lieut.-Col. R. Bodley Scott. As the aetiology and correct treatment of this relatively rare condition are controversial, it may be of value to report these three cases and then discuss the problem in the light of the available literature.

Case 1.-A soldier, aged 29 and employed as a bobbin-runner in civil life, was admitted to hospital in March, 1941, complaining of swelling of the neck and breathlessness. $\mathrm{He}$ had pneumonia twice as a child and again at the age of 25 , this last attack being severe and followed by streaking of the sputum with blood ; convalescence was slow, for he was away from work for six months, and this illness left him slightly short-winded. A year later he had another acute respiratory illness diagnosed as bronchitis, and this time he noticed a lump above the manubrium sterni and prominence of the veins of the chest wall. In September, 1939, he developed right-sided pleuritic pain which lasted for a month: subsequently this was replaced by a grating sensation on respiration which persisted intermittently until admission. In October, 1940, he joined the Army (grade two because of flat feet!), but physical training caused great breathlessness, swelling of the neck, headaches, and "spots before the eyes." His cough and pleuritic pain also recurred, and the veins on the chest wall enlarged further. He entered a military hospital, where he gained considerable temporary relief from a venesection (16 oz.) in the arm.

On examination his face appeared "puffy" and the cervical veins were grossly distended. Dilated veins were present over the anterior chest wall, especially in the region of the costal margin. Loud pleural friction was heard on the right anteriorly, and there was evidence of displacement of the mediastinum towards this side. Otherwise physical examination was negative. A chest radiograph (Fig. 1) showed thickening of the pleura on the right, displacement of the heart to this side, and slight widening of the superior mediastinum. The Wassermann reaction and Kahn test were negative, and the sputum contained no tubercle bacilli. The arm-lung circulation time by the ether method was 13 seconds (normal, 3 to 10 seconds). The blood contained 5,840,000 erythrocytes per c.mm., with a haemoglobin concentration of 100 per cent (Haldane). 
On April 17, 1941, the superior vena cava was explored by anterior thoracotomy through the second right interspace. This gave adequate although imperfect exposure. The upper lobe was covered with large tortuous vessels which converged on to a band of adhesions connecting the upper lobe to the mediastinum just above the hilum; it is not clear what part these vessels played in the collateral circulation. This band was divided between clamps in order to improve the exposure. There were no other adhesions, which is of interest in view of the long history of pleuritic pain. A hard, ill-defined mass could be palpated in the superior mediastinum. The pleura over it was incised and an attempt made to dissect the structures within the mediastinum, but the dense fibrosis completely obscured

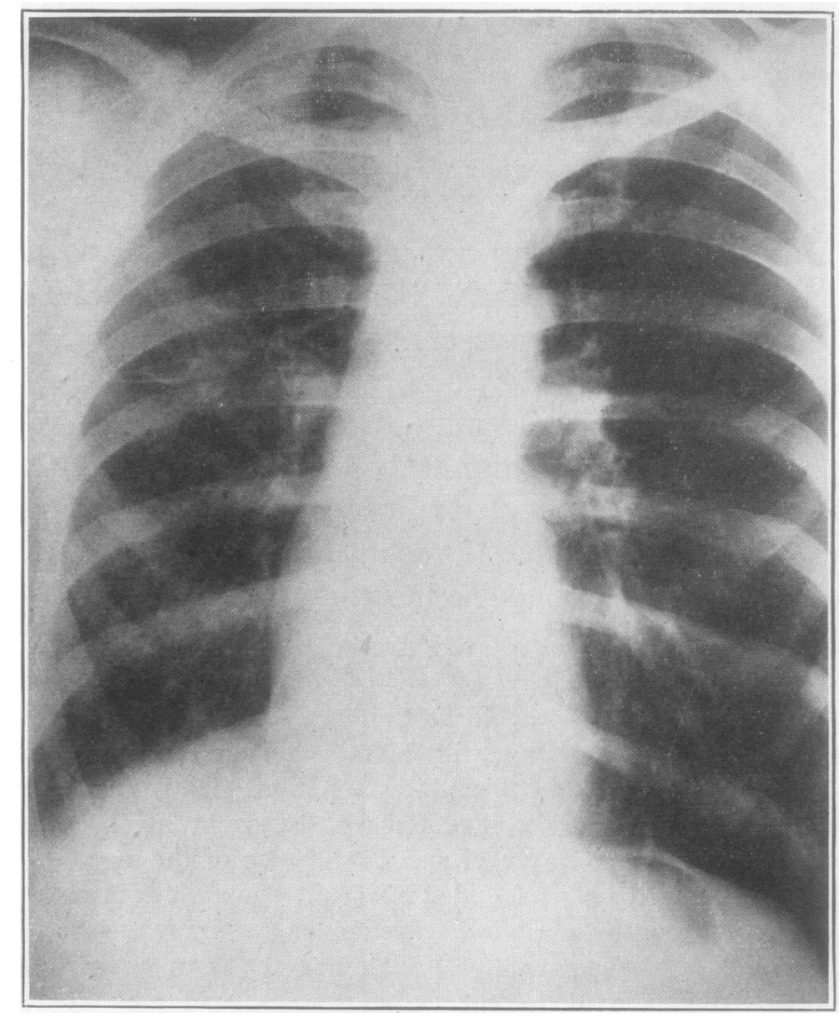

Fig. 1.

the anatomy. As further exploration was clearly most hazardous, the operation was abandoned and the chest closed.

Apart from a transient respiratory infection the operation made the patient neither better nor worse. He was discharged from the Army four months later.

He was readmitted in November, 1945. His symptoms due to venous obstruction had remained unchanged during the four-year interval, and he had been able to work as a radio-engineer. Clinically and radiologically no change could be appreciated. The venous pressure in the arm was $180 \mathrm{~mm}$. of water: after constriction of the chest with a sphygmomanometer cuff so as to obstruct the collateral circulation, the pressure exceeded $310 \mathrm{~mm}$. Although the constriction was released as soon as increased congestion of the face was 
noted, this test caused the patient acute distress due to a feeling of suffocation which he experienced immediately after removal of the sphygomanometer cuff.

Case 2.-A lorry-driver, aged 25, was admitted to hospital on Feb. 20, 1945, complaining of swelling of the face and neck. He had been in good health until 1941, when he felt

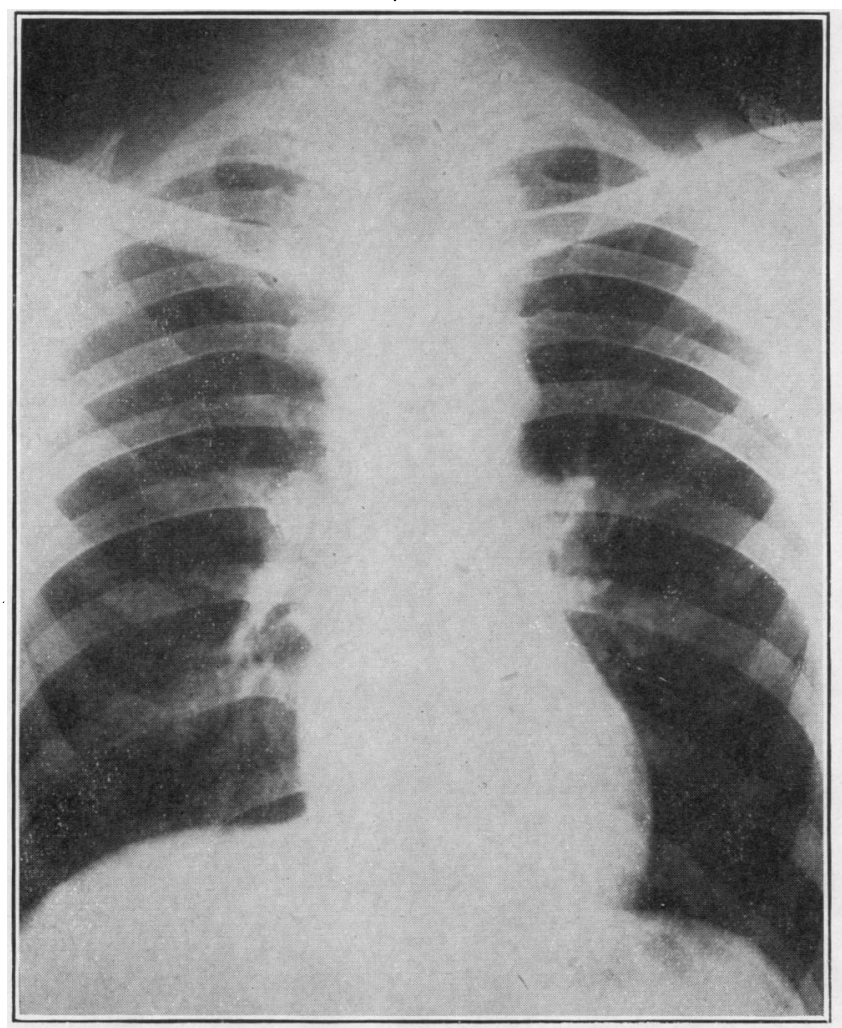

FIG. 2.

tired and coughed up a little blood-stained sputum. These symptoms resulted in his admission to a sanatorium, where nothing abnormal was discovered except for a calcified focus near the right hilum. A year later his face and neck swelled so that he could not get his collar on, and his eyes became "runny." These symptoms, however, subsided in two weeks and he kept well until a year before admission, when the swelling, most marked on the right side and worse after exercise or bending down, recurred. There were no other symptoms except slight shortness of breath.

On examination the patient presented the typical picture of superior vena caval obstruction, with swelling of the face and neck and slight cyanosis of the lips. The superficial veins in the neck were grossly distended, and those of the upper extremities slightly so. The subcutaneous veins on the front of the chest and abdomen were dilated, and the flow in these was from above downwards. No other abnormality was found.

A chest radiograph (Fig. 2) showed slight widening of the superior mediastinum and calcified glands in the upper part of the right hilum. Most regrettably no blood tests for syphilis were done. 


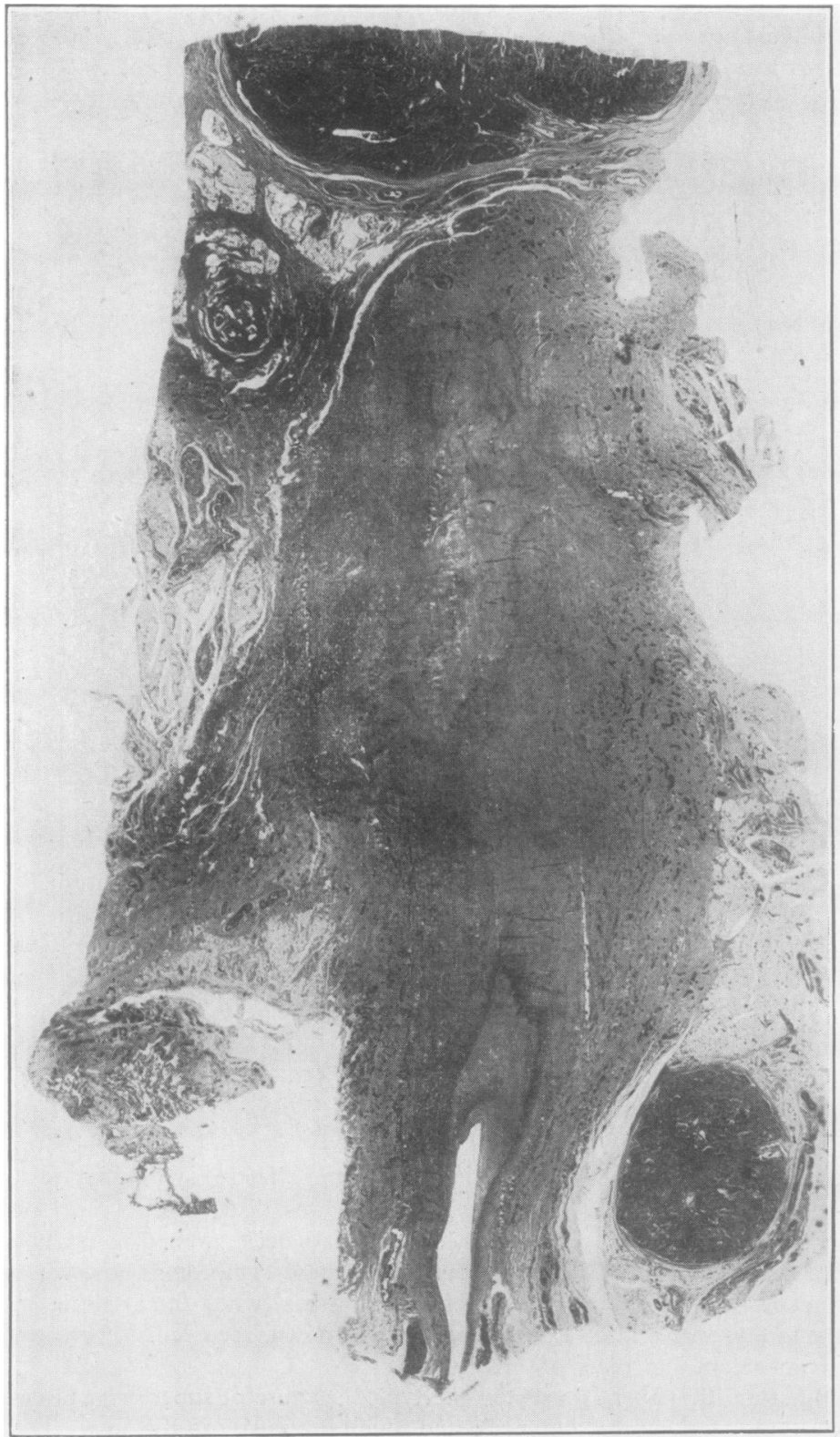

FIG. 3.-Longitudinal section through the obliterated superior vena cava. In the lower part of the section the narrowed lumen of the vein can be seen: above this the site of the vein is replaced by dense fibrous tissue. Elsewhere the mediastinal cellular tissue is replaced by vascular granulation tissue in which enlarged lymphatic glands are embedded. 
On Feb. 26, 1945, the mediastinum was explored through a postero-lateral incision in the fourth intercostal space. This approach was used in preference to an anterior incision, as it was thought that it would interfere less with the collateral circulation and would provide better exposure, as indeed it did.

Many adhesions containing large veins stretched between the cervical cupola of the pleura and the upper lobe, and the surface of the latter was covered by a prominent venous plexus. It was evident that these vessels allowed some of the brachio-cephalic venous return to enter the pulmonary circuit. A mass of dense fibrous tissue was found in the superior mediastinum, and the superior vena cava could not be recognized. The superior intercostal and azygos veins were grossly dilated, but the azygos arch was obliterated in the fibrotic mass. In view of the dense fibrosis it was thought unwise to search for the superior vena cava by sharp dissection, and the chest was therefore closed.

The neck veins were less distended after operation, and the patient stated that his face felt less swollen, presumably as a result of the blood-letting at operation. On the other hand he was restless, sometimes irrational, and more cyanosed than before. However, convalescence was free from anxiety until the ninth post-operative day, when he collapsed, passed a large, bloody stool, and vomited a small amount of blood. As the collapse was severe and persistent blood was transfused, three pints being given into the internal saphenous vein before death occurred ten hours after the onset of the collapse. When the "drip" was being set up some oedema of the ankles was noticed.

At autopsy the cellular tissue of the superior mediastinum was found to be replaced by dense but vascular fibrous tissue in which numerous much enlarged lymphatic glands were embedded. Calcified glands were found at the right hilum, but there was no caseation or calcification in those in the mediastinum. The superior vena cava was totally obliterated from the point of union of the two innominate veins for more than one inch (Fig. 3); below this an extremely narrow lumen was present. The azygos arch was completely obstructed. No abnormality was found in the heart or substance of the lungs.

The surprise came from examination of the abdomen, for the same glandular enlargement with surrounding fibrosis was found in the para-aortic region, and this had caused gross stenosis of the inferior vena cava at the level of the renal veins, distal to which a recent thrombus occluded the main vein and extended into both common iliac veins (Fig. 4).

Apart from congestion no lesion was found in the alimentary tract to account for the intestinal haemorrhage. Prof. Geoffrey Hadfield and Dr. A. G. Stansfield summed up the histoiogical picture with the statement that it suggested a chronic progressive inflammatory lesion, but there was no evidence of tuberculosis or other specific infection.

Case 3.-On July 9, 1945, a soldier, aged 34, was admitted to the Royal Herbert Hospital, Woolw.ch, under the care of Lieut.-Col. R. Bodley Scott. He complained of swelling of the neck and left arm, pain in the back, and breathlessness. He had been in good health until August, 1944, when, while in Egypt, he developed a dull aching pain across the shoulders which lasted four weeks. He was well again until November, 1944, when "lumps like marbles" appeared under his chin and he was admitted to hospital, where all his teeth were extracted and the "lumps" slowly disappeared. After about three months in hospital the backache recurred, and a radiograph of the chest was taken, but this showed no abnormality. The backache increased in severity, and he returned to the United Kingdom in April, 1945. When he was admitted to the Royal Herbert Hospital in July he complained, in addition to the pain, of a dry cough, breathlessness, orthopnoea, and swelling of the neck and left arm, with a bursting sensation in the head if he bent down. He gave a past history of having had balanitis in December, 1940, but no recognized venereal disease. 


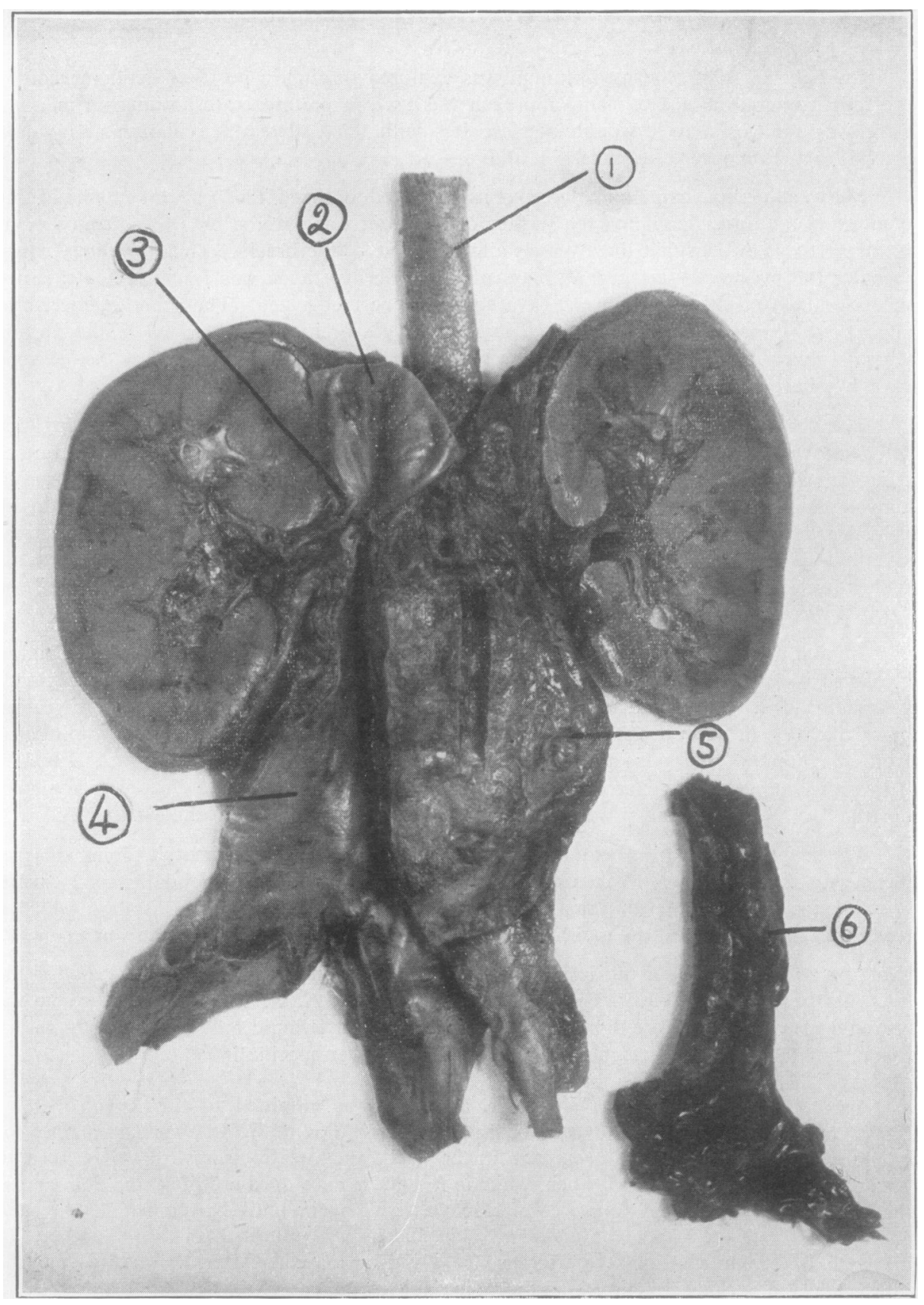

Fig. 4.-Photograph of retroperitoneal structures removed at autopsy from Case 2, showing (1) abdominal aorta ; (2) upper unobstructed part of the inferior vena cava ; (3) extreme narrowing of the inferior vena cava at the level of the renal veins; (4) lower part of the inferior vena cava, which was considerably narrowed by the matted mass (5) of fibrous tissue and enlarged lymphatic glands surrounding the aorta; (6) recent thrombus removed from (4). 
On examination there was swelling of the face and left arm with gross distension of the veins of these regions, but no other clinical abnormality was detected. A chest radiograph (Fig. 5) showed a high right diaphragm and slight, ill-defined widening of the superior mediastinum, but no tumour was demonstrable. The Wassermann and Kahn reactions of both the patient and his wife, but not of his three children, were positive.

He was treated with sodium iodide, but this had to be discontinued owing to iodine sensitivity; he was subsequently given bismuth and penicillin, and their administration was followed by considerable relief of his symptoms.

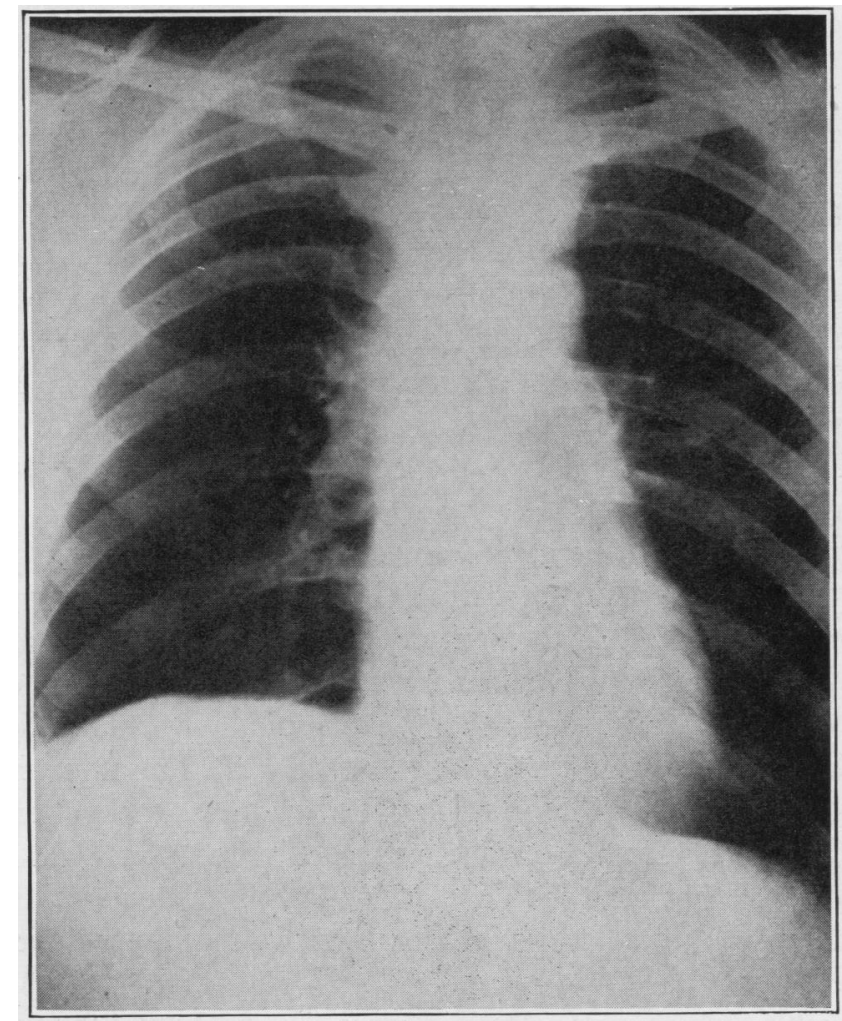

Fig. 5 .

By October, 1945, he was less breathless and the cough had cleared up. He still experienced a feeling of pressure in the neck on bending down; he required three or four pillows at night, and even then awoke with a stiff feeling in the face.

On examination the face appeared "full" and slightly cyanosed. There was puffy, nonpitting swelling of the root of the neck, with gross enlargement of the superficial veins. The arms were not swollen, and the pulse and blood pressure $(134 / 80)$ on the two sides were equal. The veins over the anterior chest wall and in the flanks were distended. The Wassermann reaction remained positive.

This case was diagnosed as gummatous mediastinitis with superior vena caval obstruction. 


\section{Discussion}

The clinical picture requires little comment, for it is simply that of superior vena caval obstruction, usually of gradual onset, for which no adequate explanation, e.g., tumour or aneurysm, can be discovered. The condition is to be distinguished from central spread of a simple thrombo-phlebitis arising in the main veins of the upper extremities, as the history does not start with swelling of an upper limb. In all cases in which the obstruction is limited to the superior vena cava, whatever the cause, it is noteworthy that the collateral venous return, which has been fully described by Blasingame (1938) and demonstrated by venography by Zeman (1945), is far more adequate for the upper extremities than for the head and neck: hence there is frequently no swelling of the upper limbs in an established case.

The symptoms are said to be more severe when the superior vena cava is cbstructed on the cardiac side of the entrance of the azygos vein (Carlson, 1934); and Hinshaw and Rutledge (1942) claim that the site of obstruction can be determined clinically by the fact that enlarged veins extending over the costal margin on to the abdominal wall are seen only when the azygos is included in the obstruction. Under the latter conditions right-sided pleural effusion may occur, but Case 2, reported above, disproves Cleland's (1941) suggestion that effusion always follows.

The venous pressure in the area drained by the superior vena cava is raised, and Hitzig (1942) has demonstrated that it rises further on the application of a tourniquet to the chest so as to interfere with the collateral circulation. This test, which is unnecessary for diagnosis, may cause the patient much discomfort (as described under Case 2) ; the rise in venous pressure is not relieved immediately the thoracic compression is removed, so that, if the tourniquet is not released until there is an increased sensation of facial fullness, the patient may suffer immediately afterwards from a terrible feeling of suffocation.

There is little doubt that the circulation time is prolonged: in Case 1 the arm-to-lung circulation time of 13 seconds is above the upper limit of normality (10 seconds).

Polycythaemia, which was noted in Case 1, has been reported on several occasions.

As far as I know, Case 2 is unique in that the same chronic inflammatory process involved the retroperitoneal cellular tissue and finally caused thrombosis of the inferior vena cava. The scar tissue appeared to be distributed in relation to the para-aortic glands and to have compressed the aorta and inferior vena cava secondarily. It is interesting to consider why the thrombosis caused intestinal haemorrhage. Berblinger (1942) claims that thrombosis of the inferior cava is usually associated with gastro-intestinal haemorrhage owing to the intense portal congestion, due to much of the systemic blood attempting to return to the heart through communications with the portal system. 


\section{Aetiology}

Ochsner and Dixon (1936) were able to find only 28 cases of superior vena caval thrombosis due to chronic mediastinitis reported in the literature. Of these 28,11 were stated to be due to syphilis, 10 to tuberculosis, 1 to pyogenic infection, 1 to trauma, and 5 were of unknown aetiology. Erganian and Wade (1943) state that the diagnosis of tuberculosis has frequently depended on the finding of calcified foci, and that of syphilis on positive serological findings, and they are not prepared to accept such evidence. They suggest that there is a pathological entity, "chronic fibrous mediastinitis"; but this suggestion does not get any nearer the fundamental cause of the condition. The three cases reported in this paper do not help materially in solving the problem of aetiology. It is very probable that Case 3 was due to syphilis, but it must be conceded that such a diagnosis rests largely on the positive serological findings. No clue as to the aetiology of Cases 1 and 2 was discovered.

\section{TREATMENT}

Several observers have commented on the marked, although temporary, relief given to these patients by venesection. This certainly proved true in Case 1.

Anti-syphilitic treatment is obviously indicated in those cases which are suspected of being syphilitic in origin. Case 3 undoubtedly improved clinically following such treatment, but this may have been due to the fact that a lapse of time allowed an improvement in the collateral circulation.

The problem of giving permanent relief to the non-syphilitic cases remains difficult. Gray and Skinner (1941) report three cases in which exploration was undertaken and the fibrous tissue overlying the vein incised longitudinally: two of these cases are stated to have been improved by operation, the venous pressure falling from 330 to $250 \mathrm{~mm}$. and from 416 to $290 \mathrm{~mm}$. (normal, 40 to $150 \mathrm{~mm}$. water) respectively, but the third died two days after operation. Another case treated similarly, with resulting improvement, is reported by Ochsner and Dixon (1936). These authors explain the improvement on the hypothesis that release of the cicatrix around the superior vena cava allows recanalization of the occluded vessel, but it is difficult to believe that new vessels could be opened up under the conditions found at autopsy in Case 2. In addition to these moderately successful cases there are a number of fruitless explorations on record, e.g., Cases 1 and 2 , and these exploratory procedures have carried a high mortality, which is a strong deterrent to surgical treatment unless it offers a very good chance of cure or, at least, great improvement.

The records make the prognosis of untreated cases appear bad, but this is probably due more to extension of the inflammatory process than to the obstruction itself. In this connexion Blasingame's (1938) report of a patient aged 93, in whom complete old-standing occlusion of the superior vena cava was found on dissection, is of interest. In addition there are seven cases on record (Claverie, 
1858 ; Beevor, 1894 ; Balzer, 1901 ; Comby, Vigouroux, and Collet, 1906 ; Gossage, 1912 ; Martin and others, 1934 ; Zeman, 1945) which survived for periods between 15 and 31 years; thus superior vena caval obstruction (to be distinguished from the process causing the obstruction) is in itself compatible with reasonably long life. Osler (1903) stated that with a slow sclerotic process, and even with complete obstruction of the vein, there is usually sufficient time for an adequate collateral circulation to develop, and the patient is thus able to live for a long time with but little discomfort.

I have therefore come to the conclusion that exploration of these cases is an unjustifiable procedure.

\section{SUMMARY}

Three cases of obstruction of the superior vena cava due to chronic inflammation of the superior mediastinum are described. One of these (Case 2) had a similar inflammatory process in the retroperitoneal tissues, which ultimately resulted in thrombosis of the inferior vena cava and the death of the patient.

The cause of the inflammatory process is discussed with special reference to tuberculosis and syphilis, but no final conclusion is reached.

The treatment is discussed, particularly with regard to surgical exploration. Operation is not warranted, as it has been shown to be hazardous and often, if not always, fruitless. In addition, evidence has been found in the literature indicating that the condition is compatible with a long life without undue discomfort.

\section{REFERENCES}

Balzer, E. (1901). Contribution à l'étude de l'oblitération et de la compression de la veine cave supérieure. Paris.

Beevor, H. R. (1894). Trans. med. Soc., London, 17, 332.

Berblinger, W. (1942). Schweiz. med. Wschr., 72, 869.

Blasingame, J. F. L. (1938). Arch. Pathol., 25, 361.

Carlson, H. A. (1934). Arch. Surg., Chicago, 29, 669.

Claverie, G. E. (1858). Thèse de Paris, No. 26.

Cleland, W. P. (1941). Brit. J. Tuberc., 35, 141.

Comby, A., Vigouroux, A., and Collet, G. (1906). Bull. Mém. Soc. méd. Hôp., Paris, 23, 136.

Erganian, J., and Wade, L. J. (1943). J. thorac. Surg., 12, 275.

Gossage, A. M. (1912). Proc. roy. Soc. Med., Clin. Sect., 6, 47.

Gray, H. K., and Skinner, I. C. (1941). Surg. Gynec. Obstet., 72, 923.

Hallet, C. H. (1848). Edinb. med. Surg. J., 69, 269.

Hinshaw, H. C., and Rutledge, D. I. (1942). J. Lab. clin. Med., 27, 908.

Hitzig, W. M. (1942). J. Mt. Sinai Hosp., N.Y. 8, 625.

Martin, R., Rouëssé, and Fiocconi (1934). Bull. Mém. Soc. méd. Hôp., Paris, 50, 192.

Ochsner, A., and Dixon, J. L. (1936). J. thorac. Surg., 5, 641.

Osler, W. (1903). Johns Hopk. Hosp. Bull., 14, 169.

Zeman, F. D. (1945). J. thorac. Surg., 14, 330. 